\title{
Learning Commons en bibliotecas académicas
}

\author{
Larisa González Martínez \\ Instituto Tecnológico y de Estudios Superiores de Monterrey - ITESM, México.
}

CASE REPORTS

\section{Resumen}

Como toda creación humana, las instituciones se transforman y evolucionan con el paso del tiempo. Dicho principio afecta también a las bibliotecas académicas, las cuales han debido cambiar constantemente para responder a las necesidades de sus usuarios. El espacio físico de la biblioteca académica es uno de los muchos aspectos marcados por el cambio, ejemplo de lo cual son los espacios para el trabajo colaborativo en bibliotecas académicas, mejor conocidos como Learning Commons en la literatura especializada. Este trabajo tiene como propósito exponer las características de este modelo, para lo cual se hará un breve recuento de la historia de la planeación y la construcción de las bibliotecas académicas. Asimismo, se expondrá la manera en la cual se ha implementado el esquema Learning Commons de la biblioteca del Instituto Tecnológico y de Estudios Superiores de Monterrey (ITESM), campus Monterrey en México.

Palabras clave

Aprendizaje activo ; Desarrollo de bibliotecas ; Bibliotecas académicas ; Espacios para el aprendizaje colaborativo ; Arquitectura bibliotecaria ; Diseño de bibliotecas ; Instituto Tecnológico y de Estudios Superiores de Monterrey ; México

\section{Learning Commons in Academic Libraries}

\section{Abstract}

Like all human creations, institutions transform and evolve over time. Libraries also have changed to respond the needs of its users. Academic libraries physical spaces are one of the turned aspects, an example are the Learning Commons (spaces for collaborative work in academic libraries). The main purpose of this paper is to expose the characteristics of the Learning Commons model with a brief account of the history of planning and construction of academic libraries. This paper also aims to present the manner in which a Learning Commons has been implemented at the library of Instituto Tecnológico y de Estudios Superiores de Monterrey (ITESM), Campus Monterrey in Mexico.

\section{Keywords}

Active learning ; Library development ; Academic libraries ; Learning commons ; Library architecture ; Lbrary design ; Instituto Tecnológico y de Estudios Superiores de Monterrey ; Mexico

\section{Introducción}

Al igual que otros edificios (y siguiendo un principio básico de la arquitectura), las bibliotecas académicas han determinado la disposición de sus espacios y su forma exterior en función de sus propósitos y funciones. Debido a esto, a lo largo de su historia, las bibliotecas académicas se han adaptado para dar cabida a las actividades de sus usuarios, especialmente cuando la tecnología se sumó a su trabajo cotidiano. Uno de los resultados de estos cambios ha sido el nacimiento de los espacios adaptados para el aprendizaje colaborativo, conocidos como Learning Commons en la literatura especializada. El presente artículo tiene como propósito exponer las características de 
este modelo, para lo cual se hará una breve revisión de las transformaciones que se han operado en las bibliotecas académicas y sus espacios a lo largo de su historia, además de que se desarrollará la manera en la cual este tipo de entornos académicos en bibliotecas se implementaron en la Biblioteca del Instituto Tecnológico y de Estudios Superiores de Monterrey (ITESM), campus Monterrey.

\section{Metamorfósis de las bibliotecas académicas}

La historia de la arquitectura de las bibliotecas académicas nació con el surgimiento mismo de este tipo de edificios en la Edad Media en universidades y centros de estudio como las escuelas de medicina y derecho de Salermo y Bolonia, o las universidades de París, Salamanca y Sevilla (Escolar, 1990, p. 200) entre otras no menos importantes.

La necesidad de administrar enormes colecciones impresas y de favorecer la consulta definió la estructura de estas primeras bibliotecas, la cual era muy sencilla pues estaba conformada básicamente por recintos en los cuales se podían encontrar diversos muebles para la lectura y el almacenaje de libros. Dado que la biblioteca académica universitaria era un lugar para la lectura y el estudio, se requirió una buena iluminación a través de ventanas (Gallo León, 2012) y un espacio considerable destinado al almacenamiento de textos, al estudio individual -para el cual se debía contar con un número importante de asientos para los usuarios- (Escolar, 1990, p. 204) y a la colocación de muebles adecuados para la consulta de libros, muchos de los cuales permanecían encadenados a pupitres perpendiculares a la pared (Gallo León, 2012, p. 86) a fin de evitar el robo (Muñoz Cosme, 2004, pp. 63 y 64).

La imprenta propició una mayor propagación de la cultura escrita e hizo de las bibliotecas importantes difusoras del conocimiento más allá del papel que jugaban como un símbolo de prestigio y custodias de la información (Gallo León, 2012, p. 92). Así pues, durante los siglos XVII y XVIII la construcción y planeación de bibliotecas académicas trató de evitar contingencias como los incendios y la humedad o el bullicio de las calles. Es en este momento que los espacios físicos de las bibliotecas comienzan a especializarse, por lo que sus salas son de distintas dimensiones y tamaños de acuerdo con su uso, además de estar bien comunicadas y de permitir su ampliación para la custodia de una mayor cantidad de libros. La sala de lectura individual se separa del lugar destinado al depósito de libros y se exige la colocación de estanterías no muy largas a lo largo de las paredes y, en muy contadas ocasiones, en el centro (Muñoz Cosme, 2004, pp. 207-209).

A finales del siglo XVIII y principios del siglo XIX la creación e implementación de nuevos sistemas de clasificación que facilitaron la consulta para los usuarios generó nuevos cambios en la acomodación de los libros y, por consiguiente, en los interiores de las bibliotecas académicas. Si bien anteriormente hubo muchos intentos para organizar y catalogar el conocimiento existente (Manguel, 2005, pp. 201-211), fueron Mevil Dewey con su sistema de clasificación decimal y la Biblioteca del Congreso con la propia quienes, a la par de facilitar considerablemente el acceso a la información, cambiaron también la disposición física misma de los libros, anteriormente basada en posiciones fijas en las estanterías (Lerner, 1999, pp.169-171).

Ante la demanda de creación de un número cada vez mayor de instituciones de instrucción superior, el siglo XX fue testigo del nacimiento y multiplicación de una gran cantidad de bibliotecas académicas a lo largo y a lo ancho del planeta. Lo anterior, aunado a una expansión en las áreas de investigación hizo que se crearan diversos servicios bibliotecarios como los acuerdos de adquisición cooperativa para el desarrollo de colecciones entre varias bibliotecas, los planes de preservación para materiales de poco uso, la elaboración de catálogos unificados y el préstamo interbibliotecario (Lerner, 1999, pp.171-172).

A estos cambios se sumó el uso de nuevas herramientas desde finales del siglo XIX y durante buena parte del siglo XX en las bibliotecas. Para que el conocimiento circulara y no sólo se acumulara (Bueno Vieira, 2006, p. 53), los bibliotecarios adoptaron herramientas como la máquina de escribir, el telex, el teléfono, el mimeógrafo y el fax (Bueno Vieira, 2006, p. 55). Por otra parte, con la llegada de la llamada era de la información, las bibliotecas académicas pasaron por una nueva etapa de cambios debido al auge de tecnologías como la computadora, las fichas perforadas, el disco óptico, las redes electrónicas (Bueno Vieira, 2006, p. 55) y en épocas más recientes las redes sociales y otras plataformas de comunicación virtual.

Con el advenimiento de las tecnologías y la consiguiente diversificaron de los servicios en las bibliotecas académicas se creó una amplia variedad de nuevas salas y/o secciones en sus edificios como los módulos de préstamo, la sala de publicaciones periódicas y el área de referencia. Un ejemplo de estos cambios fue el 
nacimiento de lo que en su momento fue llamado el Centro de Información, el cual posee una arquitectura y una distribución de los espacios más complejos, además de que procura flexibilizar las áreas destinadas a cumplir con las tres funciones básicas que todo edificio construido para ser una biblioteca debe llevar a cabo: los complejos administrativos, la sala de lectura y el depósito de libros (Muñoz Cosme, 2004, p. 229).

El Centro de Información es un modelo definido primordialmente en términos de sus colecciones y de sus actividades de instrucción, estas últimas centradas principalmente en la información, por su búsqueda del desarrollo de habilidades en el acceso, uso o en la evaluación de la misma (Todd, 2009) en los usuarios. Por tanto, el Centro de información forma parte de un paradigma prevaleciente en el ámbito arquitectónico bibliotecario hasta hace relativamente poco tiempo: los edificios centrados en las colecciones y los servicios (Felicié Soto, 2007, p. 1).

No obstante, si bien el manejo de tecnología es relevante para el quehacer bibliotecario actual, la mera existencia de computadoras al interior de una biblioteca no es suficiente para satisfacer las necesidades de sus usuarios (Diggs, 2009, p. 33). Partiendo de esta premisa, las bibliotecas académicas debieron reinventarse nuevamente pero, ahora también, por la influencia de dos factores del ámbito académico reciente, que de acuerdo con Laura Wernick son (Wernick, 2011):

1. La gradual desaparición del libro impreso como principal fuente de información y el aumento en el acceso a documentos únicos no impresos: Esto implica que, en la actualidad, los textos digitales y/o multimedia han dejado de ser meras copias de fuentes impresas para convertirse, en algunos casos, en una fuente original que no requiere de soporte en papel alguno.

2. Una creciente demanda de entornos sociales para el trabajo académico y de espacios destinados al trabajo colaborativo:

Durante cierto tiempo, la construcción de bibliotecas académicas no supo responder del todo a ciertas problemáticas propias de las necesidades de los usuarios. Debido a esto, últimamente el modelo ha sufrido un cambio, debido al cual los espacios físicos al interior de las bibliotecas académicas comienzan a estar más centrados en las personas que en las colecciones. De esta manera, los usuarios se han apoderado (empowerment) de los espacios físicos bibliotecarios y/o de las tecnologías y recursos de información que los complementan (Felicié Soto, 2007, p. 1). Desde este enfoque, si bien anteriormente las bibliotecas eran colecciones, ahora son conexiones, ya sea de redes de información y/o tecnologías pero, sobre todo, de individuos y de sus diversas formas de pensar (Muñoz Cosme, 2004, p. 346) generando variadas interacciones.

En este panorama no hay que perder de vista los elementos educativos que ejercen influencia en las bibliotecas académicas. Actualmente un elemento clave de los esquemas de enseñanza-aprendizaje es el constructivismo. En este paradigma los aspectos cognitivos, afectivos y sociales del comportamiento del individuo no son un mero resultado del ambiente ni de sus disposiciones internas, sino una construcción propia que se lleva a cabo día a día mediante la interacción de los factores antes mencionados (Carretero, 2009, p. 22).

Para el modelo constructivista el conocimiento es una construcción (Carretero, 2009, p. 22) que resulta de la actividad de un sujeto activo, no de la recepción pasiva, y su adquisición, es decir, la actividad cognitiva, se lleva a cabo en el mundo de la experiencia (Mata, Rodríguez Diéguez y Bolívar Botía, 2007, p. 219), por lo que el sujeto se convierte en el único responsable de su comportamiento, su pensamiento y su conocimiento (Mata, Rodríguez Diéguez y Bolívar Botía, 2007, p. 218).

Con el constructivismo, la docencia ha cambiado en las universidades y el resto de las instituciones educativas (Tarabay y Perinat, 2005, pp.86 y 87). Así pues, si bien no se elimina la clase magistral, el maestro deja de ser el protagonista en el aula y el alumno se convierte en el gestor de su propio 
conocimiento, haciendo que el proceso de enseñanza-aprendizaje se centre en el educando (Tarabay y Perinat, 2005, p.86).

Por otra parte, la evaluación del desempeño del alumno también ha variado. El examen se convirtió en evaluación continua, ya sea a través del trabajo en equipo o de investigaciones que detonen los intercambios, la discusión en grupo, el compartir información, dar forma a un texto elaborado en conjunto, etcétera (Tarabay y Perinat, 2005, pp.86 y 87).

Al panorama anterior se suman también las técnicas didácticas -como el Aprendizaje Colaborativo (AC), el Aprendizaje Basado en Problemas (ABP), el Aprendizaje Orientado a Proyectos (POL), el Método de Casos, etcétera- (Instituto Tecnológico y de Estudios Superiores de Monterrey, 2011) , para las cuales el trabajo en equipo y la búsqueda de información para la solución de problemáticas son elementos importantes en el aprendizaje.

El constructivismo y las técnicas didácticas acentuaron la dimensión social del aprendizaje, el cual se manifiesta a través del trabajo en equipo. Por lo anterior se produjo una transformación de los espacios educativos a fin de facilitar el trabajo grupal. Por ello en las bibliotecas académicas se incrementó la demanda de lugares para trabajar en equipo, pues los usuarios actuales poseen características distintas a los de antaño más habituados a leer, estudiar y escribir individualmente.

En este sentido el concepto del "nativo digital" toma especial relevancia. Según este término, un nativo digital es toda persona que nació y creció con las nuevas tecnologías de tal forma que ya son parte de su vida (Rodríguez Montiel, 2011). En términos generales, un nativo digital convive de manera natural con toda clase de dispositivos electrónicos -computadora, teléfono celular, etc.- (Rodríguez Montiel, 2011), está permanentemente conectado a la red, requiere de una gratificación inmediata (Myers y Sundaram, 2012, p. 32) etcétera. Ante este escenario, las bibliotecas académicas tienen una enorme cantidad de desafíos entre los que se encuentra la creación de "espacios inspiradores que apoyen la creatividad y la innovación; que brinden soporte a la creación de redes; que propicien el trabajo individual o la interacción en grupo" (Brindley, 2009, p.11), etcétera.

\section{Learning Commons en bibliotecas académicas}

Como una respuesta a los factores presentados en el apartado anterior, en las bibliotecas académicas ha surgido el concepto de los espacios para el aprendizaje colaborativo o Learning Commons. Para Bennet (Bennet, 2003, p. 37) este modelo pudo haber surgido en la década de los 90's, en las instituciones de educación superior, concretamente, de una sala de uso común en la cual todos los miembros de la comunidad académica podían reunirse de manera informal en torno a intereses compartidos, sobre todo después de las comidas. Con la tecnología, esta idea se renovó dando lugar al Learning Commons, como una versión actualizada de estos primeros espacios de intercambio.

El concepto Learning Commons es relativamente nuevo, por lo que su definición aún está en construcción. Así, para Felicié Soto el término Learning Commons nació de un concepto similar denominado Information Commons que es un conjunto de puntos de acceso a la red y tecnologías de la información en un contexto en el que diversos recursos (físicos, digitales, humanos y sociales) se organizan para brindar soporte al aprendizaje. Felicié Soto menciona también que un Learning Commons es el resultado de la unión de los Information Commons con iniciativas de aprendizaje de otros actores del proceso educativo (Felicié Soto, 2007, p. 6).

Por otra parte, algunos autores sostienen que el concepto Learning Commons nació de la evolución del concepto Information Commons, considerados espacios digitales "donde la gente puede reunirse y colaborar" (Bundy, 2008, p. 17). Para algunos investigadores un Learning Commons no debe ser dominado por los recursos de información (Bundy, 2008, p. 17) sino que más bien, debe de incorporar a las estrategias de enseñanza de los centros 
educativos un "conocimiento profundo" de la conducta independiente de los estudiantes y de su forma de aprender, además de un total entendimiento de las estrategias que apoyan y soportan el desarrollo de los alumnos (Bennet, 2003, p. 39) ya sea que provengan de la academia, o de otras instancias educativas. Para esto, un Learning Commons debe ser planeado teniendo en cuenta cuestiones de fondo sobre el proceso de enseñanza-aprendizaje y escuchando la voz de alumnos y profesores (Bundy, 2008, p. 17).

Un Learning Commons deberá tener la capacidad de adaptarse a los cambios frecuentes que tienen las diferentes tareas de aprendizaje de los alumnos, definidas por ellos mismos (apoderamiento -empowerment-) y no por las autoridades de la institución educativa -bibliotecarios, programadores, maestros, etc.- (Bennet, 2003, p. 39). Por otra parte, un espacio con estas características deberá favorecer el aprendizaje colaborativo (Bundy, 2008, p. 18) y proveer servicios alimentarios. Sin embargo, hay que evitar caer en el error que Sullivan llama "Bibliotecas Starbucks", cuyo único atractivo radica en el mobiliario y en los alimentos que se ofrecen, con lo que se propicia el ocio y poco se aporta al rendimiento académico de los estudiantes (Sullivan, 2012). Los servicios alimentarios no son un fin en sí mismos, por lo que se debe de procurar que estén ligados a actividades sociales y educativas en forma (Bennet, 2003, p. 39), lo que para Diggs (2009, p. 35) es sociabilizar la biblioteca, haciéndola un espacio para la cultura y un lugar que propicie experiencias de aprendizaje. Para finalizar, otra definición establece que un Learning Commons es un conjunto de espacios comunitarios de estudio que favorecen el aprendizaje colaborativo y el fácil acceso a servicios alimentarios (Wernick, 2011).

En términos generales, los estudiosos del tema sostienen que un Learning Commons está compuesto por tres tipos de elementos:

1. Físico-espaciales. El elemento más importante de la Biblioteca ya no es la información por lo que su espacio físico deja de ser un lugar destinado a la custodia de una colección (Corbett, 2011) y equipado para el trabajo individual. De esta manera, un Learning Commons debe predisponer al estudiante para el estudio a través de un espacio físico acogedor que posea las siguientes características:

- El ambiente. Con elementos como el color y la textura un Learning Commons debe de tener la capacidad de crear diferentes atmósferas al interior de la misma biblioteca. Los espacios deben de ser adecuados para estudiar y trabajar aunque también debe de haber zonas para descansar y relajarse (incluyendo áreas externas como patios y jardines). En términos generales, la biblioteca debe de poseer una estructura flexible en la cual no se sacrifique la funcionalidad por la belleza (Sullivan, 2012).

- El mobiliario. Debe de ser cómodo, con la opción de ser móvil para crear atmósferas diferentes y tener la capacidad de adecuar los espacios a las actividades que realicen los usuarios. Sin embargo, si bien los muebles son importantes, no pueden dictar las disposiciones del espacio y distraer la atención de otras actividades académicamente más relevantes (Sullivan, 2012).

- La iluminación. De preferencia se debe emplear luz natural pues la reducción en el uso de luz artificial (Sullivan, 2012) se verá reflejado en los presupuestos. En caso de que no sea posible acondicionar una iluminación natural, las luces artificiales deben estar debidamente dispuestas para ofrecer un ambiente confortable.

2. Humanos. En un mundo tecnológicamente complejo como el actual, un elemento clave deberá ser la presencia de especialistas que puedan auxiliar al usuario en el acceso y recuperación de documentos de todo tipo (Wernick, 2011).

3. Tecnológicos. Para facilitar la creación de espacios de aprendizaje interactivo (Sullivan, 2012) la tecnología debe de estar completamente integrada a un Learning Commons (Wernick, 2011). 
Actualmente se pueden encontrar numerosos ejemplos de la implementación de los Learning Commons en bibliotecas de todos los niveles educativos. Algunos casos son:

- Centros de educación básica: Beaufort Elementary School en Carolina del Sur, Estados Unidos (Beaufort Elementary School, s.f.); la R.J. Hawkey Elementary School en Alberta Canadá (Hawkey Elementary School, s/f) y la Presbyterian School en Houston, Texas, Estados Unidos (Holt, 2011).

- Educación media: Chelmsford High School en North Chelmsford, Massachusetts, Estados Unidos (Chelmsford High School, s.f.) y la Concord-Carlisle Regional High School en Boston, Estados Unidos (Cicchetti, 2010, pp. 52-58).

- Centros universitarios: North Carolina State University, Carolina del Norte, Estados Unidos (North Carolina State University, s.f.); la University of Guelph, Guelph, Ontario, Canadá (University of Guelph, 2012); la Seattle University Seattle, Washington, Estados Unidos (Seattle University, 2012) y las Universidades de Bath, Reading, Sussex, Universidad de Birckbeck, York y la Universidad de Royal Holloway en el Reino Unido (Serrano-Vicente, 2007, pp. 307-318).

\section{Learning Commons en la Biblioteca del ITESM}

Los espacios para el aprendizaje colaborativo (Learning Commons) de la Biblioteca del Instituto Tecnológico y de Estudios Superiores de Monterrey, Campus Monterrey comenzaron a gestarse a finales de los años 90's. En ese momento, la intención de las autoridades era crear un espacio en el cual las mesas, las sillas y los "muros" del mismo tuvieran ruedas para que los usuarios fueran libres de conformar espacios de estudio de acuerdo a las necesidades propias del trabajo en equipo.

Posiblemente por la falta de una conceptualización sobre el tema (en ese momento no se conocía o no se había formulado el concepto Learning Commons ni algo similar para bibliotecas académicas), el proyecto no prosperó sin embargo, ya entrado el siglo actual, se trabajó con un par de arquitectos a los cuales se volvió a insistir en la idea sin resultados alentadores.

En enero de 2011 nuevamente se elaboró una propuesta para la remodelación de la Biblioteca del Campus. Como en este momento ya existía literatura académica que planteaba la existencia y construcción de espacios orientados hacia el aprendizaje y el trabajo colaborativo (Learning Commons), la propuesta se llevó a cabo con el nombre y las características de este nuevo modelo bibliotecario. La realización parcial esta propuesta es la que hoy se tiene en el 4to. Piso, para lo cual se inició el proceso de selección de mobiliario y materiales a mediados de junio de 2011 y se inauguró en agosto de ese mismo año (González Martínez, 2012).

Las características del Learning Commons de la Biblioteca del Tecnológico de Monterrey campus Monterrey se pueden resumir en 4 elementos:

1. Información: Conformada por materiales impresos y por la biblioteca digital del campus. Por las posibilidades que la tecnología ofrece se ha implementado una herramienta de consulta para las colecciones digitales, consistente en un discovery layer, llamado apris@. Esta mejora permite realizar búsquedas unificadas en las diferentes plataformas de las bases de datos además de que auxilia también a las colecciones impresas por facilitar la búsqueda en el catálogo.

2. Tecnologías de información: Acceso a 10 a 100 Mbps de internet, computadoras, impresoras y software.

3. Expertos: El personal especializado de la biblioteca del Tecnológico de Monterrey, campus Monterrey se divide en dos categorías. En primer lugar se encuentran los bibliógrafos por área de conocimiento y/o departamento, quienes guían a los usuarios para encontrar la información que requieren, además de fortalecer el vínculo con la Academia a través de todo un programa de capacitación y formación a usuarios. 
Los bibliógrafos son auxiliados por los referencistas de la biblioteca. Asimismo, los asesores de software y hardware apoyan a los usuarios ante problemas o dificultades técnicas.

4. Ambientes de trabajo: El Learning Commons de la biblioteca del Tec campus Monterrey se compone de diversos ambientes equipados para el trabajo de un individuo o de un grupo de hasta 40 personas desarrollando diversas actividades de aprendizaje. Cabe señalar que la diferenciación de los ambientes de trabajos y la creación de atmósferas se ha logrado a través de mesas, sillas, sillones, "pintarrones" y pantallas con ruedas que pueden ser trasladados por los usuarios de acuerdo a sus necesidades, o bien, a través de mobiliario sin ruedas que forma "islas" en el Learning Commons. Cabe señalar que una de estas “islas" es una cafetería y 8 son clústeres de computadoras (González Martínez, 2012).

Si bien aún es necesario evaluar muchas variables que se transformaron con la implementación del Learning Commons en el campus Monterrey, es una realidad que el proyecto ha generado un impacto al interior de la Biblioteca y en otros centros del Tecnológico de Monterrey. Ejemplo de lo anterior es el proyecto que se tiene en las preparatorias de la Rectoría de la Zona Metropolitana de Monterrey del Tecnológico de Monterrey (Santa Catarina, Garza Sada, Garza Lagüera, Valle Alto y Cumbres) para la construcción y puesta en marcha de Learning Commons.

\section{Conclusión}

Desde una perspectiva meramente funcional se puede decir que existe una "relación simbiótica" entre la forma en la que las bibliotecas han sido construidas y la función que éstas han realizado durante su existencia (Dowlin, 2004, p. 12). Cabe señalar que esta simbiosis también ha impactado considerablemente los servicios que una biblioteca es capaz de ofrecer a sus usuarios.

El Learning Commons o espacios de aprendizaje colaborativo en bibliotecas académicas es un paso más en la historia de los centros de información académicos y su evolución, por lo que ofrece interesantes oportunidades para el estudio y análisis relacionados con su implementación, los servicios que pueden ofrecerse y las interacciones Biblioteca-Academia que se pueden llevar a cabo.

\section{Referencias}

Beaufort Elementary School. (s/f). The 21st Century Media Center-The Learning Commons. [Página de Internet]. Recuperado el 11 de Julio 2013 de http://bes.beaufortsc.schoolfusion.us/modules/cms/pages.phtml?pageid=190190\&SID

Brindley, D. J. (2009). Challenges for Great Libraries in the Age of the Digital Native. Information Services \& Use, 29(1), 3-12. doi:10.3233/ISU-2009-0594

Bueno Vieira, G. J. (2006). Impacto tecnológico y arquitectura en bibliotecas. Buenos Aires: Alfagrama.

Bundy, A. (2008). University libraries and educational change: the information literacy window of Opportunity. Biblioteca Universitaria. 11(1), 13-21. Recuperado el 29 de Julio de 2012 de http://www. redalyc.org/articulo. oa? id=28512651003

Carretero, M. (2009). Constructivismo y educación. Buenos Aires: Paidós.

Chelmsford High School. (s/f). Learning Commons. [Página de internet]. Recuperado el 12 de Julio 2013 de http://Learningcommons.chs.chelmsford. schoolfusion.us/modules/groups/integrated home. phtml? gid =1584374\&sessionid=8ae9c19804499869f4e dc439bbca92a2

Cicchetti, R. (2010). Concord-Carlisle Transitions to a Learning Commons. Teacher Librarian. 37(3), pp. 52-58.

Recuperado el 14 de Julio de 2012 de http://0-

ehis. ebscohost.com. millenium. itesm. $\mathrm{mx} / \mathrm{eds} / \mathrm{detail}$ ?vid=5\&sid=fd6674bc-34ea-4add-9b38-

c9a2b512f4f5\%40sessionmgr113\&hid=110\&bdata=Jmxhbmc9ZXMmc2l0ZT1|ZHMItbGl2ZQ $\% 3 \mathrm{~d} \% 3 \mathrm{~d} \# \mathrm{db}=\mathrm{aph} \& \mathrm{AN}=4$ $\underline{8874439}$

Diggs, V. (2009). From library to Learning Commons: A metamorphosis. Teacher Librarian. 36(4), 32-38. Recuperado el 3 de Agosto 2012 de http://biblioteca.mty.itesm.mx/mty/bds/salta bd.php?col id=pad 
Dowlin, K. E. (2004). The library as place: Challenges in the Digital Age en Marie-Françoise Bisbrouck et al. (Editores), Libraries as places: Buildings for the $21^{\text {st }}$ century Proceedings of the Thirteenth Seminar of IFLA's Library Buildings and Equipment section together with IFLA's Public Libraries Section, Paris, France, 28 July-1 August 2003, München: Saur, p. 11-37.

Escolar, H. (1990). Historia de las bibliotecas. $3^{a}$ ed. Salamanca: Madrid: Fundación Germán Sánchez Ruipérez; Madrid: Pirámide.

Evans, G.E. y Zarnosky Saponaro, M. (2005). Depeloping Library and Information Center Collections. Wesport, Connecticut: Libraries Unlimited.

Felicié Soto, A. M. (2007). Reflexión sobre el cambio de paradigma en el uso del espacio en las bibliotecas: diez líneas de acción. Acceso: Revista Puertorriqueña de Bibliotecología y Documentación. 9, 1-16. Recuperado el 23 de Agosto 2012 de http://www.redalyc.org/articulo.oa?id=25620541002

Gallo León, J. P. (2012). Forma y función de los edificios de bibliotecas universitarias: Herramientas para su evaluación. [Tesis doctoral en línea]. Murcia: Universidad de Murcia. Recuperado el 24 de Julio 2013 de http://www.tdx.cat/bitstream/handle/10803/80598/TJPGL. pdf;jsessionid=577C5B20849EAA3782D5A82C45BC2979.tdx2?sequence $=1$

Hawkey Elementary School. (s/f). About our Learning Commons-Library/Technology Area. [Página de Internet]. Recuperado el 11 de Julio 2013 de http://rjhawkey.rockyview.ab.ca/library

"Heracleitus". (2013). En Encyclopædia Britannica. Encyclopædia Britannica Online Academic Edition. Encyclopædia Britannica Inc. Recuperado el 10 de Junio 2013 de http://0www.britannica.com.millenium.itesm.mx/EBchecked/topic/262438/Heracleitus

Instituto Tecnológico y de Estudios Superiores de Monterrey. (2011). Técnicas didácticas. Presentación. [Página de internet]. Recuperado el 23 de Julio 2012 de http://sitios.itesm. mx/va/diie/tecnicasdidacticas/1 1.htm

Holt, K. (2011). Looking in on the Learning Commons. [Página de internet]. Recuperado el 11 de Julio 2013 de http://www.pshouston.org/cf news/view.cfm?newsid=46

Lerner, F. (1999). Historia de las bibliotecas del mundo: desde la invención de la escritura hasta la era de la computación. Buenos Aires: Troquel.

Manguel, A. (2005). Una historia de la lectura. México, D.F.: Joaquín Mortiz.

Mata, F. S. , Rodríguez Diéguez J.L y Bolivar Botía A. (Directores). (2007). Diccionario enciclopédico de didáctica. Vol. I. Puebla, Gileditores.

Myers, M. D. y Sundaram, D. (2012). Digital Natives. University Of Auckland Business Review, 15(1), 28-37. Recuperado el 26 de Julio 2013 de http://0-ehis.ebscohost.com. millenium. itesm.mx/eds/pdfviewer/pdfviewer?sid=1a737d58-618c-4db9-bcdfdad21f25a253\%40sessionmgr113\&vid=3\&hid=106

Muñoz Cosme, A. (2004). Los espacios del saber; Historia de la arquitectura de las bibliotecas. Gijón, Asturias: Ediciones Trea.

Presbyterian School. (s/f). A vision for a Learning Commons. [Documento en línea]. Recuperado el 11 de Julio 2013 de http://www.pshouston.org/uploaded/Academics/library/Learning Commons.pdf

Rodríguez Montiel, E. (2011). Nativos digitales en la reconfiguración radiofónica. Razón y Palabra, (76). Recuperado el 26 de Julio de 2013 de http://www.redalyc.org/articulo.oa?id=199519981014

Serrano-Vicente, R. (2007). Los Learning centers en el Reino Unido. Estudio de caso de seis universidades medianas. El Profesional De La Información. 16(4), 307-318. doi:10.3145/epi.2007.jul.04

Sullivan, M. (2011).Divine Design: How to Create the 21st-Century School Library of Your Dreams. School Library Journal. 57(4), 26-32. Recuperado el 3 de Mayo 2012 de http://biblioteca.mty.itesm.mx/mty/bds/salta bd.php?col id=pgd

Tarabay, F. y Perinat, A. (2005). En torno a la innovación docente: Competencia versus conocimiento. Compendium. 8(14), 8191. Recuperado el 8 de Julio de 2013 de http://www.redalyc.org/articulo.oa? id=88001406

Todd, R.J. (2009). There is Knowledge to be Gained. School Library Media Activities Monthly. 25(10), 55-58. Recuperado el 9 de Septiembre 2012 de http://0-ehis. ebscohost.com. millenium. itesm. mx/eds/pdfviewer/pdfviewer?vid=4\&sid=3e14bade-9383-47a4931f-32ad04b2a033\%40sessionmgr113\&hid=116 
Wernick, L. (2011). The School Library Space Is Changing. American School \& University. 83(9), 24-28. Recuperado el 1 de Agosto 2012 de http://biblioteca.mty.itesm.mx/mty/bds/salta bd.php?col id=pqd

\section{Datos de la autora}

\section{Larisa González Martínez}

Miembro del Departamento de Programas de Instrucción a Usuarios de la Dirección de Vínculo Académico y Desarrollo de Capital Humano de la Dirección de Bibliotecas del Instituto Tecnológico y de Estudios Superiores de Monterrey, campus Monterrey. Locutora del programa Para Leer en Bicicleta y promotora cultural de Frecuencia Tec 94.9 FM.

larisa.gonzalez@itesm.mx , larisa.gonzalez.martinez@hotmail.com

$\begin{array}{ll}\text { Recibido-Received } & : 2013-09-04 \\ \text { Aceptado-Accepted } & : 2013-12-30\end{array}$

\section{$(\infty)$ EY}

This work is licensed under a Creative Commons Attribution 4.0 United States License.

\section{ULIS D-Sonk}

This journal is published by the University Library System of the University of Pittsburgh as part of its D-Scribe Digital Publishing Program and is cosponsored by the University of Pittsburgh Press. 